'Pulmonary and Critical Care Medicine Brigham and Women's Hospital Harvard Medical School Boston, MA. ${ }^{2}$ Anesthesia and Intensive Care, Sant'Andrea Hospital, ASL VC, Vercelli, Italy. ${ }^{3}$ Lab of Respiratory Disease, Department of Clinical and Experimental Medicine, Katholieke Universiteit Leuven, Leuven, Belgium. ${ }^{4}$ Department of Pulmonology Semmelweis University, Budapest, Hungary. ${ }^{5}$ Department of Clinical Science, University of Bergen, Bergen, Norway. ${ }^{6}$ Airway Disease Infection Section. National Heart and Lung Institute. Imperial College London, London, UK. 'Department of Pneumology and Critical Care Medicine, Thoraxklinik, University Hospital Heidelberg, Heidelberg, Germany. ${ }^{8}$ Department of Pharmacology and Toxicology, Maastricht University, Maastricht, The Netherlands. ${ }^{9}$ Department of Pneumology, School of Medicine in Katowice, Medical University of Silesia, Katowice, Poland. ${ }^{10}$ Institute of Occupational Medicine and Environmental Health, Sosnowiec, Poland.

\title{
Juniors' voice at the ERS International Congress, Amsterdam 2015
}

\section{Juniors' voice}

This year's ERS International Congress was, as always, well-organised, providing participants with a good mixture of translational and clinical science. The emerging trend throughout the conference was the personalised approach, dedicated to better understanding, characterising and thus managing patients as individuals with unique combinations of clinical characteristics rather than as a single diagnostic entity.

One hot topic discussed was the concept of comorbidity networks, which is especially important in chronic diseases, including chronic obstructive pulmonary disease (COPD), accompanied by a wide range of extra-pulmonary clinical manifestations affecting the outcome or treatment. Targeting not only the disease but also its co-morbidity network will be the next step towards personalised medicine. This new paradigm has led to the development of the new concept of "network science" to better understand complex diseases by mapping the interconnectivity of co-morbidities.

Another important clinical item, which is also highly relevant in terms of epidemiology, was the overlap between COPD, asthma and cancer. While the overlap between COPD and cancer has previously been acknowledged, asthma-COPD overlap syndrome (ACOS), is only recently gaining increasing attention. Although a consistent definition is still lacking, ACOS is generally defined as a condition with clinical features from both asthma and COPD. Consequently, extensive phenotyping should take place prior to including ACOS patients into clinical trials in order to clarify whether a strict definition will be beneficial for routine clinical practice. To date, clinicians assess these patients every day, as they probably represent $15-45 \%$ of the entire COPD population, but do not diagnose or treat them differently than pure COPD or asthma patients as there are no dedicated guidelines. Their lack is partly due to the fact that only a few studies have assessed treating these patients, as most COPD studies exclude nonsmokers and patients with some bronchodilator reversibility, whereas asthma studies excluded the opposite groups. Patients with ACOS require different management to patients with COPD alone but, if treated optimally, have a better prognosis. This ACOS symposium exemplified the emergence of phenotypes and overlap syndromes in chronic airway disorders, highlighting the need of large-scale studies to
Cite as: Polverino F, Longhini F, Vanaudenaerde B, et al. Juniors' voice at the ERS International Congress, Amsterdam 2015. Breathe 2015: 11: 303-305. 
better understand the molecular and genetic background of airway diseases. One possible approach for this includes systems biology, which investigates diseases at numerous end points in a very sophisticated and unbiased statistical manner. During the congress, the first results of the recently completed pan-European study U-BIOPRED (Unbiased Biomarkers in Prediction of Respiratory Disease Outcomes) were presented. This systems medicine approach is also applied within the field of lung transplantations by means of large cohorts including the SysCLAD (Systems Prediction of Chronic Lung Allograft Dysfunction) cohort. Indeed, the lung transplant field embraces the awareness that such systems medicine studies together with larger patient cohorts and multicentre approaches are essential to obtaining solid results. A special recognition should go the work of David Ruttens who performed a first European-wide study with over 5000 lung transplant patients from 13 European lung transplant centres, which was also covered in a press release by ERS.

The importance of phenotyping and subtyping chronic lung diseases was further underling by the discussed shift in paradigm observed within the asthma field. Nowadays, asthma is considered a collection of a wide range of temporary alternating phenotypes. More importantly, airway inflammation is no longer an obligate component of bronchial asthma definition. The Global Initiative for Asthma (GINA) suggests maximising the understanding of the underlying mechanisms leading to asthmatic symptoms in each individual. Therefore, underused techniques to measure airway inflammation or forced oscillation technique, which may be especially useful in diagnosing patients with poor cooperation during pulmonary function tests performance.

In the last few years, personalised medicine has also entered the field of lung transplantation, especially regarding phenotyping chronic lung allograft dysfunction (CLAD). Understanding the two major CLAD entities, bronchiolitis obliterans syndrome and restrictive allograft syndrome, and how to deal with them with respect to diagnosis, follow-up, therapy and outcome is again a major concern.

Besides characterisation of various clinical phenotypes, the influence of smoking on the immune mechanisms underlying different disease phenotypes was discussed. It was shown that the development of an emphysema-COPD phenotype or lung cancer in smokers depends on which cell types are affected. This was exemplified by a process called T-cell exhaustion, which is characterised by stepwise and progressive loss of T-cell functions and can culminate in the physical deletion of the responding cells mainly in chronic inflammatory diseases as well as in tumor expansion. The influence of pre- and postnatal secondhand smoke exposure on the development of lung pathologies was also discussed, as evidence strongly links such exposure to a wide range of manifestations, including extra-pulmonary pathologies, in the offspring.

Latent tuberculosis (TB) infection was also highlighted in a "Hot Topic" symposium following the recent publication of the WHO guidelines [1] on this subject. Approximately one-third of the world's population is infected with latent TB and the new guidelines provide a public health approach to identification and treatment of individuals with highest risk of progression to active disease including immunosuppressed patients or immigrants from high TB burden countries [1]. Taking into account the vast influx of refugees into Europe, a special interest was raised regarding newest developments in diagnosis and treatment of TB and multidrug-resistant TB. Rates of TB are thus expected to continue to increase as more people gain access to European healthcare systems.

Much interest was also generated by presentations from the EMBARC (European Bronchiectasis Registry) study group, a new ERS pan-European collaborative registry of patients with non-cystic fibrosis bronchiectasis [2]. Key questions that will be tackled by EMBARC include the role of Pseudomonas spp. eradication, type and duration of antibiotic therapy in exacerbations and identification of risk factors for disease progression. There is heterogeneity in bronchiectasis service provision within Europe at present and EMBARC will provide guidance for future standardisation.

As always, there was an important update on patients with critical conditions. High-flow oxygen through nasal cannula (HFONC) has been one of the most discussed new topics in critically ill patients, especially its use in the treatment of hypoxaemic acute respiratory failure. The FLORALI (High-FLow Oxygen Therapy for the Resuscitation of Acute Lung Injury) study, showed that HFONC reduced overall mortality as well as the cumulative incidence of intubation in specified subset of patients [3]. Although some concerns were raised regarding the noninvasive ventilation settings applied in this study, its results surely created awareness regarding the use of noninvasive ventilation in hypoxaemic acute respiratory failure.

Another rising topic within the treatment of patients with critical conditions is the use of extracorporeal $\mathrm{CO}_{2}$ removal. Recently, veno-venous systems with an external pump have been introduced to efficiently and safely reduce endotracheal intubation in patients with acute chronic respiratory failure.

The recent Volkswagen scandal with fraudulent emission data, obtained in laboratory testing of the engines rather than real-life measurements of air-pollution, was also discussed. This has led to the following statement issued by the ERS Executive Committee: "Assessments of air pollution impacts on health and ecosystems should be based on real-world emission data, not on values obtained in laboratory settings, which have 
become more and more remote from the real world over recent years."

There was also an excellent programme of sleep medicine presented. In particular the symposium on neurological and memory issues in sleep disordered breathing presented new and exciting advances in the field. More difficult, were the disappointing outcomes of the SERVE-HF trial which showed increased mortality in patients with central sleep apnoea in the context of low ejection fractions $(<30 \%)$ using adaptive servo-ventilation therapy. This was an unexpected outcome overall and one which will require a great deal of soulsearching in the next few months. Something for all who treat sleep-disordered breathing should definitely be aware of!

Alongside clinical and scientific sessions, this year's Congress again offered educational sessions, including one that allowed live observation of endoscopic procedures performed by experts in the field of interventional endoscopy in patients with lung cancer, interstitial and pleural diseases as well as emphysema and severe asthma. This unique opportunity gave members with an interest in this field the possibility of widening their knowledge and skill set.

Finally, to celebrate the benefits of exercise for health and wellbeing, Assembly 10 ran a competition that encouraged all members to track their steps each day with the aim of walking more than 10,000 steps per conference day. Dr Klaus Polke managed the most steps in a single day, an impressive 69,011! Congratulations!

Overall, this year's ERS International Congress once more has proven to be the place where science, clinic education and lifestyle meet. Indeed, not only the most recent scientific and clinical data regarding disease phenotyping, treatment modalities and environmental risks were presented, but educating our respiratory experts and pursuing a healthy lifestyle were proactively advocated as well. Therefore, we would like to invite especially those juniors who have never attended the ERS International Congress before, to join us in London next year.

\section{References}

1. World Health Organization. Guidelines on the management of latent tuberculosis infection. Available from www.who. int/tb/publications/ltbi_document_page/en/ Data last accessed 14 October 2015 .
2. EMBARC The European Bronchiectasis Registry www. bronchiectasis.eu/ Data last accessed 14 October 2015.

3. Frat JP, Thille AW, Mercat A, et al. High-flow oxygen through nasal cannula in acute hypoxemic respiratory failure. $N$ Engl J Med 2015; 372: 2185-2196. 